\title{
AVERAGING THE SUM OF DIGITS FUNCTION TO AN EVEN BASE
}

\author{
by D. M. E. FOSTER
}

(Received 31st July 1990)

\begin{abstract}
For a fixed integer $q \geqq 2$, every positive integer $k=\sum_{r \geqq 0} a_{r}(q, k) q^{\prime}$ where each $a_{r}(q, k) \in\{0,1,2, \ldots, q-1\}$. The sum of digits function $\alpha(q, k)=\sum_{r \geq 0} a_{r}(q, k)$ behaves rather erratically but on averaging has a uniform behaviour. In particular if $A(q, n)=\sum_{k=1}^{n-1} \alpha(q, k)$, where $n>1$, then it is well known that $A(q, n) \sim \frac{1}{2}((q-1) / \log q) n \log n$ as $n \rightarrow \infty$. For even values of $q$, a lower bound is now given for the difference $\frac{1}{2} S(q, n)=A(q, n)-\frac{1}{2}(q-1)[\log n / \log q] n$, where $[\log n / \log q]$ denotes the greatest integer $\leqq \log n / \log q$, complementing an earlier result for odd values of $q$.
\end{abstract}

1980 Mathematics subject classification (1985 Revision). 11 A.

As is well known, if $q \geqq 2$ is a fixed integer then every positive integer $k$ has a unique (finite) expression in scale $q$ of the form

$$
k=\sum_{r=0}^{\infty} a_{r}(q, k) q^{r} \quad \text { where } \quad a_{r}(q, k) \in\{0,1,2, \ldots, q-1\}
$$

The sum of digits function

$$
\alpha(q, k)=\sum_{r=0}^{\infty} a_{r}(q, k)
$$

and for $n>1$ its sum

$$
A(q, n)=\sum_{k=1}^{n-1} \alpha(q, k)
$$

In 1940, Bush [1] proved the best possible asymptotic result, namely that

$$
A(q, n) \sim \frac{\frac{1}{2}(q-1)}{\log q} n \log n \quad \text { as } \quad n \rightarrow \infty
$$

and since then more precise estimates for the behaviour of $A(q, n)$ and related functions 
have been obtained. In a recent paper in this journal [3] several references are given, including one to a wide ranging survey by Stolarsky [4]. In [3] a lower bound was obtained for

$$
\frac{1}{2} S(q, n)=A(q, n)-\frac{1}{2}(q-1)\left[\frac{\log n}{\log q}\right] n
$$

for all odd values of $q \geqq 9$. (As usual, $[\log n / \log q]$ denotes the greatest integer less than or equal to $\log n / \log q$ ). Since then an analogous result for all even values of $q \geqq 6$ has been found. The smaller values of $q$ yield more precise inequalities (see [2]). In fact these lower bounds complement the upper bound, namely

$$
\frac{S(q, n)}{n}<q-1
$$

for all integers $q \geqq 2$ obtained in [2].

The strategy of the proof does not depend on the parity of $q$, but when $q$ is even, the critical case takes one of two different forms and this difference is reflected in establishing the required inequality. The rather lengthy proof for odd values of $q$ was explained in some detail in [3]. However, using a slight modification of the ideas used there, we now indicate an easier method for even $q$. With this in mind it is now better to keep the original digits notation, thereby allowing for possible zero coefficients. Then every positive integer $n$ is of the form $n=n_{m}$ where

$$
n_{m}=a_{0}+a_{1} q+a_{2} q^{2}+\cdots+a_{m} q^{m}
$$

for some $m \in \mathbf{N} \cup\{0\}$ and with coefficients $a_{0}, a_{1}, \ldots, a_{m} \in\{0,1,2, \ldots, q-1\}$ and $a_{m} \neq 0$. In addition, for $1 \leqq i \leqq m-1$ we introduce

$$
n_{-1}=0, n_{0}=a_{0} \text { and } n_{i}=a_{0}+a_{1} q+\cdots+a_{i} q^{i}
$$

and, as described in [2], we have

$$
S\left(q, n_{m}\right)=\sum_{r=0}^{m}\left[a_{r}\left(a_{r}-1\right) q^{r}+\left(2 a_{r}-q+1\right) n_{r-1}\right] .
$$

We define $\beta_{q}$ as the unique even integer satisfying

$$
3 q-\sqrt{8 q^{2}-9 q+1} \leqq \beta_{q} \leqq 3 q-\sqrt{8 q^{2}-9 q+1}+2
$$

and note that equality cannot occur since $8 q^{2}-9 q+1$ is odd when $q$ is even. (For odd values of $q, \beta_{q}$ was the odd integer defined by the same inequalities, uniquely determined 
except when $8 q^{2}-9 q+1$ is a perfect square.) In the course of the derivation of the lower bound for $S\left(q, n_{m}\right) / n_{m}$ two cases arise according as

(i) $\left(3 q+1-\beta_{q}\right)^{2} q<8 q^{3}-9 q^{2}+2$

or (ii) $\left(3 q+1-\beta_{q}\right)^{2} q>8 q^{3}-9 q^{2}+2$,

and it is this fact which makes the details of the proof twice as long as in the case of odd $q$.

Numerical evidence by my colleague Mrs M. F. McCall for $5 \leqq q \leqq 13$ has brought to light the critical cases, and hence the choice of $\beta_{q}$. In case (i), when $n_{m}$ is of the form

$$
\begin{gathered}
n_{m}^{*}=n_{m-l}+\frac{1}{2}(q-2) q^{m-l+1}+\frac{1}{2} q \cdot q^{m-l+2}+\frac{1}{2}(q-2) q^{m-l+3}+\frac{1}{2} q \cdot q^{m-l+4}+\ldots \\
+\frac{1}{2}(q-2) q^{m-3}+\frac{1}{2} q \cdot q^{m-2}+\frac{1}{2}\left(q-\beta_{q}\right) q^{m-1}+q^{m}
\end{gathered}
$$

for $l \equiv 0(\bmod 2)$ it can be seen that

$$
\frac{S\left(q, n_{m}^{*}\right)}{n_{m}^{*}} \rightarrow-h_{q} \text { as } m \rightarrow \infty \text { and } m-l \text { remains fixed, }
$$

where $h_{q}=\alpha_{q}+1$ with

$$
2 \alpha_{q}=\frac{q^{2}(q-8)-\left(\beta_{q}-4\right)^{2} q-\left(\beta_{q}-2\right)\left(\beta_{q}-8\right)}{3 q^{2}+4 q+2-\beta_{q}(q+1)}
$$

Similarly the critical form for $n_{m}=n_{m}^{*}$ in case (ii) occurs when $l \equiv 0(\bmod 2)$ and

$$
\begin{gathered}
n_{m}^{*}=n_{m-l}+\frac{1}{2} q \cdot q^{m-l+1}+\frac{1}{2}(q-2) q^{m-l+2}+\frac{1}{2} q \cdot q^{m-l+3}+\frac{1}{2}(q-2) q^{m-l+4} \\
+\cdots+\frac{1}{2} q \cdot q^{m-3}+\frac{1}{2}(q-2) q^{m-2}+\frac{1}{2}\left(q-\beta_{q}\right) q^{m-1}+q^{m}
\end{gathered}
$$

Once again,

$$
\frac{S\left(q, n_{m}^{*}\right)}{n_{m}^{*}} \rightarrow-h_{q} \text { as } m \rightarrow \infty \text { and } m-l \text { remains fixed, }
$$

where $h_{q}=\alpha_{q}+1$, as before, but now

$$
2 \alpha_{q}=\frac{q^{2}(q-8)-\left(\beta_{q}-4\right)^{2} q-\beta_{q}\left(\beta_{q}-6\right)}{3 q^{2}+4 q-\beta_{q}(q+1)}
$$

Theorem. For all even $q \geqq 6,\left(S\left(q, n_{m}\right) / n_{m}\right)>-h_{q}$. When $q=2$ or 4 there is a more precise result, namely 


$$
\frac{S\left(q, n_{m}\right)}{n_{m}} \geqq-h_{q}(m) \text { where } h_{2}(m)=\frac{2\left(2^{2 m}-1\right)}{13.2^{2 m}-1}
$$

(see [2]) and

$$
h_{4}(m)= \begin{cases}\frac{9.4^{m}-6}{23.4^{m}-2} & \text { if } m \text { is odd, } \\ \frac{9.4^{m}-4}{23.4^{m}+2} & \text { if } m(>0) \text { is even. }\end{cases}
$$

The sequence of even integers $\left(\beta_{q}\right)$ increases with $q$, as does $\alpha_{q}$, and starts off as follows:

$$
\beta_{q}= \begin{cases}4 \text { if } q=6(\text { case (i)), } & 8,10,12(\text { case (ii)) } \\ 6 \text { if } q=14,16,18(\text { case (i)), } & 20,22,24(\text { case (ii)) } \\ 8 \text { if } q=26,28,30(\text { case (i)), } & 32,34,36(\text { case (ii)). }\end{cases}
$$

The sequence $\left(\alpha_{q}\right)$, correct to two decimal places, begins with

$$
\begin{gathered}
\left(\alpha_{6} ; \alpha_{8}, \alpha_{10}, \alpha_{12}\right)=(-0.30 ; 0.02,0.35,0.68) \\
\left(\alpha_{14}, \alpha_{16}, \alpha_{18} ; \alpha_{20} \alpha_{22}, \alpha_{24}\right)=(1.01,1.36,1.70 ; 2.05,2.39,2.72),
\end{gathered}
$$

and

$$
\left(\alpha_{26}, \alpha_{28}, \alpha_{30} ; \alpha_{32} \alpha_{34}, \alpha_{36}\right)=(3.06,3.41,3.75 ; 4.10,4.44,4.78)
$$

In Lemma 2.1 of [3], we proved that $3 \leqq \beta_{q}-\alpha_{q} \leqq 5$, and the same inequality holds for even $q$. More precisely, a routine calculation gives

$$
4<\beta_{q}-\alpha_{q}<5 \text { in case (i) }
$$

and

$$
3<\beta_{q}-\alpha_{q}<4 \text { in case (ii), }
$$

inequalities which, once again, are useful in the later stages of the proof of the theorem. Lemma 2.2 of [3] is no longer needed, but Lemma 2.3 needs to be adapted because of the two different values of $\alpha_{q}$ when $q$ is even. As in [3], for $2 \leqq l \leqq m+1$, we introduce

$$
F_{m}(l-1)=a_{m}\left(a_{m}+\alpha_{q}\right) q^{m}+\sum_{r=m-l+1}^{m-1} a_{r}\left\{\sum_{s=r+1}^{m}\left[2 a_{s}-q+1\right]+a_{r}+\alpha_{q}\right\} q^{r}
$$

and, once again 


$$
h(x)=x\left(x+3+\alpha_{q}-\beta_{q}\right), \quad k(x)=x\left(x+4+\alpha_{q}-\beta_{q}\right)
$$

and, in addition,

$$
p(x)=x\left(x+5+\alpha_{q}-\beta_{q}\right)
$$

and

$$
u(x)=x\left(x+3+\alpha_{q}\right)
$$

When $l=m+1, F_{m}(m)=S\left(q, n_{m}\right)+h_{q} n_{m}$ and the theorem will follow if we can find a simple form for $F_{m}(m)$ and then prove that it is positive. Using the ideas of [3], substitutions for $a_{m-1}, a_{m-2}, \ldots$ once again are chosen to reflect the critical case in which the sequence $\left(\delta_{n}\right)$, to be defined, begins with a run of zeros. Thus, for $2 \leqq r \leqq m$ and for those values of $q$ in case (i), we introduce

$$
2 a_{m}=2+2 \delta_{0}, 2 a_{m-1}=q-\beta_{q}-2 \delta_{0}+2 \delta_{1} \quad \text { and } \quad 2 a_{m-r}= \begin{cases}q-2 \delta_{r-1}+2 \delta_{r} & (r \text { even }) \\ q-2-2 \delta_{r-1}+2 \delta_{r} & (r \text { odd })\end{cases}
$$

When $l=2$, it may be verified directly that

$$
4 F_{m}(1) q^{-(m-1)}=4(q-1) u\left(\delta_{0}\right)+4 h\left(\delta_{1}\right)+\left(\frac{q+2}{q+1}\right)\left(q-8-2 \alpha_{q}+2 \beta_{q}\right)
$$

Using induction on $l$ we see that, for $3 \leqq l \leqq m+1$,

$$
\begin{aligned}
4 F_{m}(l-1) q^{-(m-l+1)} \\
=4(q-1)\left\{q^{l-2} u\left(\delta_{0}\right)+q^{l-3} k\left(\delta_{1}\right)+q^{l-4} p\left(\delta_{2}\right)+q^{l-5} k\left(\delta_{3}\right)+q^{l-6} p\left(\delta_{4}\right)+\cdots+p\left(\delta_{l-2}\right)\right\} \\
\quad+4 h\left(\delta_{l-1}\right)+\left(\frac{q+2}{q+1}\right)\left(q-8-2 \alpha_{q}+2 \beta_{q}\right)
\end{aligned}
$$

if $l$ is even, whereas

$$
\begin{aligned}
& 4 F_{m}(l-1) q^{-(m-l+1)} \\
& =4(q-1)\left\{q^{l-2} u\left(\delta_{0}\right)+q^{l-3} k\left(\delta_{1}\right)+q^{l-4} p\left(\delta_{2}\right)+q^{l-5} k\left(\delta_{3}\right)+q^{l-6} p\left(\delta_{4}\right)+\cdots+k\left(\delta_{l-2}\right)\right\} \\
& \quad+4 k\left(\delta_{l-1}\right)+\frac{q}{q+1}\left(q-8-2 \alpha_{q}+2 \beta_{q}\right)
\end{aligned}
$$


if $l$ is odd.

Now, for $2 \leqq r \leqq m$ and for those values of $q$ in case (ii), we introduce

$$
2 a_{m}=2+2 \delta_{0}, 2 a_{m-1}=q-\beta_{q}-2 \delta_{0}+2 \delta_{1} \quad \text { and } \quad 2 a_{m-r}= \begin{cases}q-2-2 \delta_{r-1}+2 \delta_{r} & (r \text { even }) \\ q-2 \delta_{r-1}+2 \delta_{r} & (r \text { odd })\end{cases}
$$

Once again an inductive proof leads to

$$
4 F_{m}(1) q^{-(m-1)}=4(q-1) u\left(\delta_{0}\right)+4 h\left(\delta_{1}\right)+\frac{q}{q+1}\left(q-6-2 \alpha_{q}+2 \beta_{q}\right)
$$

and, for $3 \leqq l \leqq m$,

$$
\begin{aligned}
& 4 F_{m}(l-1) q^{-(m-l+1)} \\
& =4(q-1)\left\{q^{l-2} u\left(\delta_{0}\right)+q^{l-3} k\left(\delta_{1}\right)+q^{l-4} h\left(\delta_{2}\right)+q^{l-5} k\left(\delta_{3}\right)+q^{l-6} h\left(\delta_{4}\right)+\ldots+h\left(\delta_{l-2}\right)\right\} \\
& \quad+4 h\left(\delta_{l-1}\right)+\frac{q}{q+1}\left(q-6-2 \alpha_{q}+2 \beta_{q}\right)
\end{aligned}
$$

if $l$ is even, whereas

$$
\begin{aligned}
& 4 F_{m}(l-1) q^{-(m-l+1)}= \\
& \quad 4(q-1)\left\{q^{l-2} u\left(\delta_{0}\right)+q^{l-3} k\left(\delta_{1}\right)+q^{l-4} h\left(\delta_{2}\right)+q^{l-5} k\left(\delta_{3}\right)+q^{l-6} h\left(\delta_{4}\right)+\cdots+k\left(\delta_{l-2}\right)\right\} \\
& \quad+4 \delta_{l-1}\left(\delta_{l-1}+2+\alpha_{q}-\beta_{q}\right)+\left(\frac{q+2}{q+1}\right)\left(q-6-2 \alpha_{q}+2 \beta_{q}\right)
\end{aligned}
$$

if $l$ is odd.

It is now an easy matter to see that $F_{m}(m)>0$ in each of cases (i) and (ii). Since $\delta_{0} \geqq 0$ and $3+\alpha_{q}>0$ for all $q \geqq 6, u\left(\delta_{0}\right)$ has a minimum value when $\delta_{0}=0$ giving $u\left(\delta_{0}\right) \geqq 0$.

In case (i) with $4<\beta_{q}-\alpha_{q}<5, h\left(\delta_{l-1}\right)$ has a minimum value when $\delta_{l-1}=1$ and accordingly

$$
\begin{aligned}
& 4 h\left(\delta_{l-1}\right)+\left(\frac{q+2}{q+1}\right)\left(q-8-2 \alpha_{q}+2 \beta_{q}\right) \geqq 4\left(4+\alpha_{q}-\beta_{q}\right)+\left(\frac{q+2}{q+1}\right)\left(q-8-2 \alpha_{q}+2 \beta_{q}\right) \\
& =\frac{q}{q+1}\left\{q+10-2\left(\beta_{q}-\alpha_{q}\right)\right\}>\frac{q^{2}}{q+1}>0 .
\end{aligned}
$$


Furthermore each of $k\left(\delta_{i}\right)$ and $p\left(\delta_{i}\right)$ has a minimum (zero) value when $\delta_{i}=0$, and in addition $q-8-2 \alpha_{q}+2 \beta_{q}>q>0$. Consequently $F_{m}(l-1)>0$ for $2 \leqq l \leqq m+1$.

In case (ii), with $3<\beta_{q}-\alpha_{q}<4$, it is clear that $u\left(\delta_{0}\right) \geqq 0, h\left(\delta_{i}\right) \geqq h(0)=0$ and $k\left(\delta_{i}\right) \geqq$ $k(0)=0$ together with $q-6-2 \alpha_{q}+2 \beta_{q}>q>0$. Also $\delta_{l-1}\left(\delta_{l-1}+2+\alpha_{q}-\beta_{q}\right)$ has a minimum value when $\delta_{l-1}=1$, in which case we find that

$$
\delta_{l-1}\left(\delta_{l-1}+2+\alpha_{q}-\beta_{q}\right)+\left(\frac{q+2}{q+1}\right)\left(q-6-2 \alpha_{q}+2 \beta_{q}\right)=\frac{q}{q+1}\left(q+8-2\left(\beta_{q}-\alpha_{q}\right)\right)>\frac{q^{2}}{q+1}>0
$$

It follows again that $F_{m}(l-1)>0$ for $2 \leqq l \leqq m+1$.

For odd values of $q$, referring back to the earlier paper [3] and once again changing to the original notation with all the exponents $t_{i}=1$, a similar modification of Lemma 2.3 can be obtained to deduce the theorem without any of the later stages of the proof. More precisely, all we have to do is to put in the additional substitution $2 a_{m}=2+2 \delta_{0}$ together with $2 a_{m-1}=q-\beta_{q}-2 \delta_{0}+2 \delta_{1}, 2 a_{m-2}=q-1-2 \delta_{1}+2 \delta_{2}$ and so on. Then the inductive method of that lemma goes through much as before to yield

$$
4 T\left(q, n_{m}\right)=4(q-1)\left\{q^{m-1} \delta_{0}\left(\delta_{0}+3+\alpha_{q}\right)+\sum_{s=1}^{m-1} q^{m-1-s} k\left(\delta_{s}\right)\right\}+4 h\left(\delta_{m}\right)+q-7-2 \alpha_{q}+2 \beta_{q}
$$

which is easily seen to be positive.

\section{REFERENCES}

1. L. E. Bush, An asymptotic formula for the average sums of the digits of integers, Amer Math. Monthly 47 (1940), 154-156.

2. D. M. E. Foster, Estimates for a remainder term associated with the sum of digits function, Glasgow Math. J. 29 (1987), 109-129.

3. D. M. E. Foster, A lower bound for a remainder term associated with the sum of digits function, Proc. Edinburgh Math. Soc. 34 (1991), 121-142.

4. K. B. Stolarsky, Power and exponential sums of digital sums related to binomial coeflicient parity, SIAM J. Appl. Math. 32 (1977), 717-730.

Mathematical Institute

UNIVERSITY OF ST ANDREWS

North HaUGH

St ANDREwS KY16 9SS 